\title{
Actualización:
}

\section{Pacientes con síntomas somáticos no explicables}

Augusto Granel

\begin{abstract}
Resumen
Se describe la problemática de los pacientes con síntomas somáticos no explicables en el ámbito de la atención primaria: síndrome de intestino irritable, dispepsia no ulcerosa, prostatodinia, dolor pelviano crónico, fibromialgia, síndrome de hiperventilación, cefalea tensional, acúfenos, globus faríngeo, tinnitus idiopático, dolor precordial atípico, dolor lumbar crónico, síndrome de fatiga crónica. Se discuten las limitaciones de las categorizaciones clínicas sobre la base de criterios diagnósticos y algunas dificultades habituales relacionadas con el paradigma de la dicotomía mente-cuerpo, que suelen conducir al intento de descartar primero lo orgánico antes de realizar el abordaje terapéutico, con el consiguiente riesgo de resultados falsamente positivos, el incremento de la ansiedad del paciente y el refuerzo del ciclo síntoma físico-consulta-prueba diagnóstica.

Se describen algunas herramientas de abordaje que se sustentan en el objetivo de aliviar al paciente más que en curarlo y el fortalecimiento de los cuidados longitudinales: programación de visitas frecuentes, pesquisa de comorbilidades tratables como depresión/ansiedad, trabajo colaborativo con el equipo de salud mental, utilización antidepresivos, utilización muy racional de las interconsultas, etc.
\end{abstract}

Granel A. Pacientes con síntomas somáticos no explicables. Evid. actual. pract. ambul. 9(6); 176-180. Nov-Dic.2006.

\section{Introducción}

La consulta de pacientes con (SS) sin una explicación clínica definida es frecuente en atención primaria. Muchos pacientes presentan estos síntomas incidentalmente pero otros lo padecen crónicamente y persisten mucho tiempo buscando atención médica. En repetidas ocasiones los SS en estos pacientes representan la expresión de dificultades emocionales que resumen complejas interacciones psicológicas, vitales, familiares y sociales. Este escenario clínico ha sido denominado de varias formas: somatizaciones, cuadros funcionales, síntomas físicos sin explicación médica, etc. Aplicado a estos sujetos, el modelo biomédico obtiene pobres resultados, ya que el mismo se basa en reconocer síntomas y generar diagnósticos, trasladando atribuciones subjetivas de los pacientes a correlatos objetivos que configuran una entidad o síndrome objetivo y específico. Sin embargo, si no se encuentran causas objetivas que puedan explicar los síntomas que describen los pacientes, aparecen grandes dificultades. Se generan mecanismos recursivos en los que los pacientes consultan ávidamente buscando una enfermedad que explique la causa de sus síntomas, a través del paradigma del modelo biomédico tradicional que implicará la realización de estudios o inclusive el tratamiento de enfermedades no existentes.

La alta utilización de recursos expresada a través de la solicitud de estudios innecesarios e interconsultas a especialistas genera altos $\operatorname{costos}^{1}$ y riesgo de complicaciones iatrogénicas. La insistencia en buscar el origen de los síntomas y la imposibilidad de calzar con un molde diagnóstico, sumado a la falta de reconocimiento del origen psicosocial genera una sensación de inefectividad en el profesional de la salud, frustración e inclusive antipatía mientras los pacientes no alivian su sufrimiento ni mejoran su incapacidad. Este artículo incursiona en la caracterización clínica, en las herramientas diagnósticas y en las bases conceptuales para el manejo y el tratamiento de los pacientes con somatizaciones.

\section{Epidemiología}

Se estima que la problemática de los SS sin explicación clínica representa el 15 al $25 \%$ de las consultas en atención primaria y que 30 a $70 \%$ de este tipo de consultas permanece sin explicación luego de una evaluación ${ }^{2}$.

Los pacientes somatizadores sufren discapacidades y disminuyen su calidad vida en forma similar a quienes padecen enfermedades crónicas graves o trastornos del ánimo como ansiedad o depresión, con la desventaja que son más persistentes y refractarios al tratamiento médico ${ }^{3}$.

\section{Categorización}

Han sido descriptas tres formas para categorizar los posibles cuadros clínicos:

- Síndromes clínicos en relación a los síntomas.

Categorías psiquiátricas

- Visión integradora.
Síndromes clínicos relacionados a los síntomas

Una primera alternativa es categorizar a estos pacientes desde el punto de vista de la descripción de síntomas definidos que van configurando patrones clínicos (ver cuadro 1) para los que cada especialidad describe sus síndromes en relación al órgano, aparato o sistema de su incumbencia.

Si bien hay muchos descriptos, no ofrecen al médico y al paciente más que una definición o etiqueta, lo que puede ser útil y estratégico en algunos casos. Sin embargo la explicación que ofrecen es limitada, y no ayudan a establecer la relación entre los motivos de consulta y los problemas psicosociales relacionados ${ }^{4}$. Además existe gran superposición de cuadros y los pacientes pueden cumplir criterios de varios síndromes al mismo tiempo ${ }^{5}$. Por ejemplo, muchos pacientes con síndrome de intestino irritable también cumplen criterios para dolor pelviano crónico o fibromialgia, y viceversa. El pronóstico de los cuadros es muy variable siendo similares los factores emocionales relacionados y las respuestas a los tratamiento. Los nombres de los síndromes parecen tener un nive de certeza biomédica o científica mucho mayor al que en realidad tienen. Si bien este tipo de visión es correcta desde un punto de vista descriptivo, revela un artificio de la especialización médica, con una tendencia a objetivar las diferencias y no las similitudes.

Cuadro 1: síndromes funcionales somáticos descriptos por especialidad.

- Gastroenterología
- Urología
- Reumatología
- Neumonología
- Neurología
- Otorrinolaringología
- Cardiología
- Ortopedia
- Miscelánea

Colon irritable, dispepsia no ulcerosa. Prostatodinia, dolor pelviano crónico. Fibromialgia.

Síndrome de hiperventilación.

Cefalea tensional, acúfenos.

Globus faríngeo, tinnitus idiopático.

Dolor precordial atípico.

Dolor lumbar crónico.

Síndrome de fatiga crónica.

\section{Categorías psiquiátricas}

La segunda forma de categorizar a estos pacientes es intentar incluirlos dentro de las categorías psiquiátricas del Manual Diagnóstico y Estadístico de Trastornos Mentales (en inglés DSM-IV) que incluye los trastornos somatomorfos ${ }^{6}$. Este consenso define estos trastornos como la presencia de síntomas físicos que sugieren una enfermedad médica y que no pueden explicarse completamente por la presencia de una enfermedad, efectos de una substancia u otro trastorno mental. Además, los síntomas deben producir malestar clínicamente significativo o deterioro social, laboral, o de otras áreas importantes de la actividad del individuo. Las subcategorías de estos trastornos son los desordenes conversivos, hipocondríacos, el trastorno dismórfico corporal, los desordenes de dolor, y la categoría residual de trastornos somatomorfos indiferenciados y no especificados. Ver cuadro 2.

El valor de esta visión ofrece la ventaja de correlacionar los síntomas psíquicos con los somáticos y pensarlos como patologías potencialmente tratables como la depresión y la ansiedad, siendo importante en este sentido, hacer diagnóstico de los componentes 
hipocondríaco y dismórfico corporal cuya respuesta al tratamiento psicofarmacológico parece ser diferente. Además, permite la identificación del subgrupo de pacientes con desórdenes de somatización, que poseen síntomas múltiples y crónicos y cuya respuesta al tratamiento es muy pobre. La desventaja de esta visión es que deja afuera muchos SS del espectro más simple, como aquellos adaptativos a situaciones especiales transitorias que representan el espectro mayor en atención primaria.

Los diagnósticos deben conllevar también estrategias de trabajo y tratamiento. Por ejemplo, raramente a un paciente que cumple criterios para síndrome de intestino irritable, el médico le diga que posee un trastorno somatomorfo indiferenciado.

Cuadro 2: criterios diagnósticos de trastornos somatomorfos.

\section{Trastorno de somatización}

Síntomas o quejas físicas de años de duración, desde antes de los 30 años de edad, que implican disfunción social, laboral u otras, y todas las siguientes manifestaciones en algún momento durante la evolución del trastorno: 1) cuatro síntomas dolorosos; 2) dos síntomas gastrointestinales; 3) un síntoma sexual; 4) un síntoma pseudo-neurológico.

\section{Trastorno de somatización indiferenciado}

Uno o más síntomas físicos que implican disfunción social, laboral u otra, con un mínimo de seis meses de evolución sin que se cumplan los criterios del trastorno de somatización.

\section{Trastorno conversivo}

Síntomas o disfunciones no explicadas de las funciones motoras voluntarias o sensoriales que sugieren un problema neurológico y se asocian con factores psicológicos.

\section{Trastorno por dolor}

Dolor en uno o más lugares del cuerpo, que predomina como motivo de consulta y se asocia con factores psicológicos.

\section{Hipocondría}

Preocupación y temor de tener una enfermedad seria basada en malas atribuciones de síntomas o funciones corporales.

\section{Trastorno dismórfico corporal}

Preocupación por algún defecto imaginario o exagerado en el aspecto físico.

\section{Desorden somatomorfo no especificado por los anteriores}

Sintomas somatomorfos que no reúnen criterios para otros desordenes somatomorfos y de menos de seis meses de evolución.

Adaptado del Manual Diagnostico y Estadístico de los Trastornos Mentales, Cuarta Edición 1994.

\section{Visión integradora}

Una tercera forma de categorizar esta problemática integra las clasificaciones anteriores en forma práctica y con fines pragmáticos. Ver figura 1. Esta parte de la premisa de que la somatización no es una entidad específica y sí un proceso con diferentes aristas y grados de expresión ${ }^{7}$ y de que si el proceso de somatización es identificado, el manejo de las diferentes formas de expresión es similar. En ocasiones puede ser más claro y conveniente utilizar las "etiquetas" los cuadros clínicos y en otras, las de la terminología psiquiátrica. Sin embargo, el profesional debe recordar que se trata de variantes de un mismo síndrome somático general ${ }^{8}$.

El espectro del síndrome es tan variable que puede relacionarse, en un extremo del espectro, con síntomas comunes o habituales como lumbalgia, cefalea o diarrea, en el contexto, por ejemplo, de una crisis vital normativa como casamiento, duelo o nacimiento de un hijo; y en el otro extremo, con síntomas crónicos, múltiples e incapacitantes que generan gran interferencia en la vida del sujeto ${ }^{9}$ y fatiga en el equipo de salud.
Figura 1: síndromes de somatización frecuentes en atención primaria.

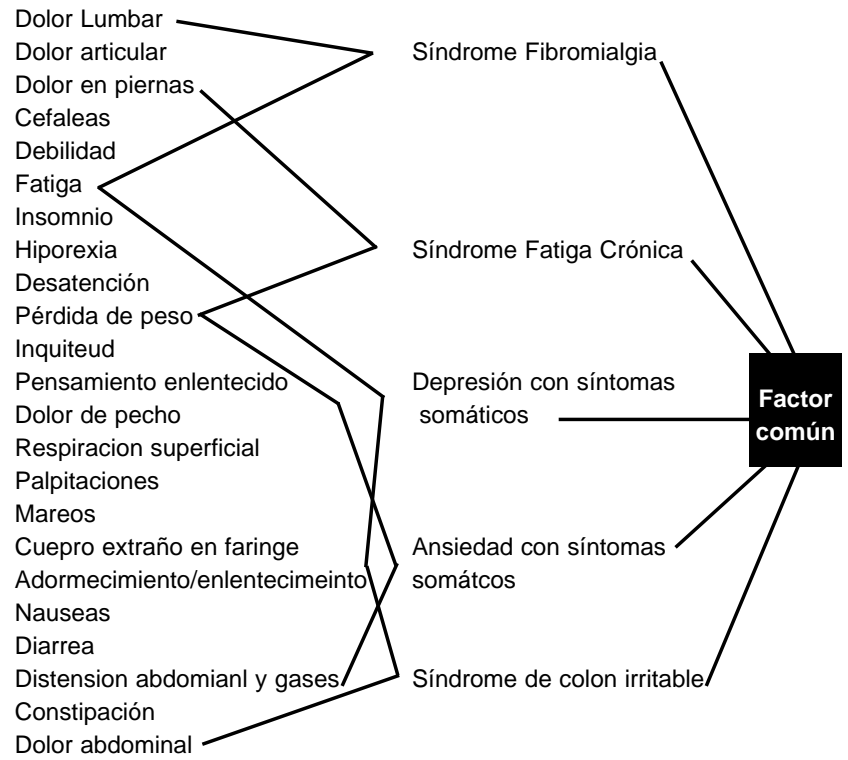

Adaptado de Deary, en el artículo original figuran los coeficientes de correlación entre las distintas variables para los tres niveles explicativos

\section{Diagnóstico}

Cuando se trabaja exclusivamente con el modelo biomédico el diagnóstico de somatización suele ser el resultado final de un largo proceso de descarte, lo que resulta costoso y frustrante para médicos y pacientes. Por el contrario, resaltamos la utilidad de hacer los diagnósticos positivamente cuando los componentes de este tipo de trastorno están presentes ${ }^{10}$.

Respecto del los criterios diagnósticos del DSM IV no suele ser muy útil su uso ya que esta problemática se comporta más como un proceso que como entidades específicas. La experiencia y la investigación clínica muestran que es posible hacer diagnóstico de somatización cuando se cumplen dos criterios que incluyen la presencia durante tiempo prolongado de tres o más síntomas vagos y desproporcionados ${ }^{11}$. Ver cuadro 3 . A menudo los pacientes tienen antecedentes de múltiples consultas y estudios, siendo esta también una variable en relación a la probabilidad de somatización.

Cuadro 3: manifestaciones clínicas sugestivas de somatización - De acuerdo a los patrones habituales de enfermedad son desproporcionadas la severidad, la duración y la repercusión emocional y social de las manifestaciones clínicas.

- No logra identificarse una causa orgánica.

\section{Sistemática de estudio}

Dada la multiplicidad de los cuadros, la investigación de los pacientes que sugieren somatización habitualmente requiere varias consultas. Una evaluación sistemática ayuda a disminuir la cantidad de consultas posteriores y a evitar perder el foco del problema.

\section{Correlación con patologías psiquiátricas}

Los trastornos psiquiátricos tienen una muy fuerte asociación con síndromes de somatización. En atención primaria el 50 a $70 \%$ de los trastornos psiquiátricos se presenta en forma somática y el $75 \%$ de los pacientes con depresión mayor o ataques de pánico que consultan en este ámbito, lo hace exclusivamente por SS $^{9}{ }^{12}$. Por ejemplo, un estudio multicéntrico realizado en varios países ${ }^{13}$, evaluó la relación entre SS y depresión en atención primaria y encontró que 45 a $95 \%$ de pacientes con depresión mayor reportaba únicamente SS.

Es importante poder diferenciar si los SS poseen un componente hipocondríaco. Habitualmente se utilizan estos términos como 
sinónimos y existe superposición, sin embargo de acuerdo a la magnitud de clínica pueden significar fenómenos diferentes.

El paciente con hipocondría siente preocupación o miedo a tener una enfermedad, y la búsqueda de consultas y análisis sirve en forma temporaria para reducir esta ansiedad. Este tipo de comportamiento se asemeja al de los pacientes con un trastorno obsesivo. El paciente con trastorno de somatización consulta por molestias derivadas de los síntomas y no por su significado.

\section{Diagnósticos diferenciales}

Diferenciar los procesos de somatización de las "causas orgánicas" puede ser muy difícil. Teniendo en cuenta la alta prevalencia de las somatizaciones, muchos expertos sostienen que es erróneo intentar una evaluación que intente descartar primero lo orgánico, para luego considerar aspectos psicológicos, sugiriendo que este proceso se lleve adelante simultáneamente ${ }^{49}$.

Cuando se evalúa pacientes con probables somatizaciones, el médico debe considerar patologías cuya aparición clínica es vaga y multisistémica. Al considerar síntomas neurológicos como parestesias o debilidad y dependiendo del contexto, es mucho más probable una somatización que formas raras de esclerosis múltiple, síndromes miasténicos o síndromes paraneoplásicos. Otro clásico ejemplo es el de aquellos síntomas neurológicos múltiples que de ser orgánicos correlacionarían con síndromes alternos de muy rara aparición. Desde ya, siempre debe primar el criterio clínico, y no será lo mismo la evaluación un paciente con dolor de pecho que haga pensar en enfermedad cororonaria, ya que los pacientes con síntomas funcionales tienen igual riesgo de enfermarse que el resto de la población y la enfermedad coronaria también es la primera causa de muerte en este grupo.

El médico se enfrenta con la ansiedad de equivocarse y el no reconocer esta sensación puede llevarlo a practicar medicina defensiva. Muchas veces, para no perder la perspectiva puede ser útil consultar con un colega con experiencia en el ámbito de la atención primaria antes que realizar una consulta con un especialista que por su sesgo tenderá a profundizar estudios y evaluaciones.

\section{Historia del sujeto, ciclos vitales, genograma y factores precipi- $\underline{\text { tantes }}$}

Es importante repasar la historia médica del sujeto con el objetivo de no perder antecedentes patológicos muy importantes, y dado que muchos de estos pacientes tienen antecedentes de policonsultas, no persistir en soluciones ya intentadas que fracasaron y que aumentarán la ansiedad del paciente y la frustración del médico.

La historia también debe ser repasada poniendo atención en sus ciclos vitales, lo que nos dará la pautas de estresores psicosociales, actuales o pasados. Es importante ser cuidadoso en este punto ya que estos pacientes tienen una mayor frecuencia de antecedentes de abuso sexual en la infancia ${ }^{14}$.

La realización de un genograma tiene en ese sentido una gran jerarquía, ya que el solo hecho de indagar en sus relaciones sin referirse explícitamente a sus síntomas, moviliza al paciente aunque éste niegue actualmente cualquier conexión con problemas psicosociales $^{15}$

Es una premisa de la evaluación no repetir estudios previos con el único objetivo de tranquilizar momentáneamente al paciente y disminuir la angustia del médico.

\section{Actitud hacia los síntomas}

Es importante ser cuidados en la evaluación y el manejo de la forma de referirse a los síntomas. Los pacientes piensan y entienden cosas diferentes de lo que los profesionales de la salud quieren expresar, lo que es particularmente cierto en estos casos ${ }^{16}$. Debe evitarse confrontar con el paciente, evitando caer en la tentación de utilizar frases como las siguientes:

"...todo está bien, el problema no es físico...;...usted no tiene nada...;....usted está más sano que yo..."

Muchos pacientes toman este tipo de frase como una acusación de faltar a la verdad, se enojan y experimentan desamparo; lo que intensificará la motivación a seguir buscando por otro lado una causa biomédica para sus síntomas. Además, desde el punto de vista biomédico tampoco tiene sentido la afirmación, ya que los síntomas son verdaderamente físicos.

Por el contrario, recomendamos que el acercamiento a la problemática por la que demanda el paciente (en este caso sus síntomas) se base en la intención de transmitirle que lo entendemos y comprendemos en su sufrimiento, con el objetivo de fortalecer la empatía y el vínculo. Pueden ser útiles frases como: "...entiendo como le afectan sus molestias, podemos seguir trabajando juntos y eso le a ayudará sentirse mejor..."

\section{Examen físico y estudios de laboratorio}

El examen físico es importante para descartar causas orgánicas y para reasegurar al paciente. Puede ser útil repetirlo en cada consulta, aunque sea en forma mínima y con el objetivo de que el paciente se sienta "revisado".

Es recomendable ser conservador con la solicitud de exámenes complementarios, que si bien pueden servir como reaseguro, conducen al aumento del riesgo de que se obtengan resultados falsamente positivos, probabilidad que aumenta en función del número de pruebas solicitadas. Recordamos que el valor predictivo positivo (VPP) de una prueba depende de la prevalencia de la condición clínica que se está pesquisando, por lo tanto el VPP tenderá a ser bajo cuando se están solicitando pruebas diagnósticas para "descartar" diagnósticos poco probables. Además, toda solicitud hecha para "descartar" hace sospechar al paciente que el médico piensa algo que no dice y refuerza el mecanismo de los síntomas. Cuando el profesional decida que es preferible negociar con el paciente o con la familia la solicitud de algún examen complementario, con el único objetivo de mostrar que no se están escatimando los recursos disponibles y de que se está intentando cuidar al paciente, consideramos que es importante explicitar previamente que se lo está haciendo con la finalidad de "dejarlo tranquilo a él (no al médico)" y que realmente pensamos que los estudios van a tener resultados normales o negativos.

\section{Tratamiento}

Bases conceptuales para el manejo basadas en la concepción biopsicosocial y colaborativa

Manejar estos pacientes con una concepción puramente biomédica puede resultar en fracasos y conlleva riesgo de producir más daño que beneficios.

La concepción bio-psicosocial ayuda a entender la totalidad del sujeto y sus relaciones. Se parte de un todo en el que existen distintos niveles interrelacionados, desde lo emocional, vincular, social y biológico ${ }^{17}$. La experiencia del paciente siempre es única y subjetiva, siendo la tarea del equipo de salud comprender su contexto. El modelo biomédico tradicional emplea la estrategia de "descartar causas orgánicas" antes de explorar otras dimensiones, lo que lleva implícito el concepto de que la enfermedad orgánica posee una importancia primaria y que los fenómenos físicos y mentales tienen fronteras muy delimitadas. En contraste, se sabe que la expresión de las emociones es reflejo de representaciones físicas y mentales que están modeladas por pautas biológicas, de la historia del sujeto, familiares y culturales ${ }^{7}$. Las emociones se expresan simultáneamente en un nivel emocional y en un nivel físico. La forma en que se diagnostica o etiqueta un paciente influye directamente en su comportamiento. Si se aplica una concepción biomédica se fortalecerán los mecanismos de formación de los síntomas y la rigidez en las manifestaciones. Si se sigue buscando el origen del problema insistiendo en soluciones ya fracasadas, esto llevará recurrentemente a mayor incapacidad y sufrimiento.

El diagnóstico debe tener entonces una utilización estratégica que ayude, por un lado, a entender que tiene "algo" y por otro, que su 
sufrimiento es reconocido. La concepción bio-psico-social incorpora además la noción de longitudinalidad. En todo paciente con cualquier patología, la historia de vida y los impactos que producen las enfermedades son únicos. Una relación médico paciente que persita en el tiempo es el mejor reaseguro contra el sobre o subtratamiento ya que aprovecha la historia del sujeto y sus aspectos relacionales. Según este enfoque, las somatizaciones son padecimientos más que enfermedades; por lo tanto el manejo debe basarse en el alivio de los síntomas y no en la cura de los mismos. El modelo colaborativo implica integrar las distintas especialidades, para trabajar en la interacción entre los distintos componentes de los complejos sistemas de salud. Los especialistas, psiquiatras y psicoterapeutas no deberían trabajar en compartimientos estancos, ya que su trabajo implica integrar lo emocional con lo somático. Por lo tanto, estos especialistas deberían trabajar en conjunto y en forma coordinada con el equipo de salud de atención primaria. Existen algunas experiencias en las que los medico de familia y los psicoterapeutas actúan en el mismo ámbito, inclusive actuando como co-terapeutas en las entrevistas ${ }^{18}$. Ver cuadro 4.

Cuadro 4: fundamentos de la concepción biopsicosocial

- Toda enfermedad afecta al sujeto en varias dimensiones o niveles (emocional, biológico, social).

- Es normal que las emociones se expresen a través del cuerpo.

- Los diagnósticos modelan a los pacientes y no sólo a los médicos.

- El tratamiento debe basarse en el alivio y en el cuidado más que en la cura y en la búsqueda de la causa.

- El seguimiento longitudinal es parte fundamental del tratamiento.

\section{Manejo Inicial}

El manejo va a depender del tiempo de evolución, de la intensidad de los síntomas y de la relación con los estresores.

El médico de atención primaria es el eje de la atención. Es probable que si lo síntomas no son muy intensos, puedan ser manejados exclusivamente con diferentes estrategias dentro de este nivel. Ver cuadro 5.

Cuadro 5: intervenciones que puede implementar el médico de atención primaria en pacientes con somatizaciones e hipocondría. - Concertar visitas frecuentes.

- Validar los síntomas.

- Establecer el objetivo de cuidar al paciente y no de curarlo.

- Mantener una actitud conservadora respecto de las interconsultas y los estudios.

Frente a los pacientes con cuadros más severos o refractarios se puede ampliar el marco, incorporando la consulta con un psicoterapeuta y/o psiquiatra. Estas consultas deben tener el concepto de ser amplificadoras del manejo del médico de atención primaria, recordando que muchos profesionales de la salud mental también están entrampados en la dualidad mente-cuerpo y es común escuchar frases como "...primero habría que descartar 10 clínico/orgánico y luego lo veo...".

\section{Estrategias de manejo en atención primaria}

El primer objetivo es poder discutir la problemática del paciente sobre la base de consultas planificadas y seguidas. En este sentido hay que acordar con el paciente que es necesario que asista cada 15 o 20 días en forma reglada y que evite concurrir a guardias u otras consultas no programadas por el médico. Se propondrán metas de trabajo que puedan ser cumplibles, siendo la herramienta terapéutica más poderosa, una buena relación médico-paciente sostenida en el contexto de una relación empática. Muchas veces no es necesario "hacer algo", simplemente hay que "estar allí", siendo el vínculo lo que logra el cambio y la resignificación de los síntomas, y evita la perpetuación de los mismos y su amplificación. Durante estas entevistas el médico de atención primaria trabajará sobre la empatía con el objetivo de ir sacando el foco en lo somático, para ponerlo en el contexto de lo psicosocial ${ }^{19}$. El médico de atención primaria dispone del recurso de formular preguntas abiertas y circulares. Esta forma de entrevistar libera información "latente" en la persona y en la familia. El poder pensar a otras personas en relación a ellos mismos permite a los individuos dar su propio sentido a su situación, observándose de una forma diferente, lo que le permite un gran poder terapéutico. Existen marcos para realizar entrevistas como el de Liderman y col., que resultan de utilidad y fueron aplicados en distintos ámbitos de atención primaria ${ }^{20}$.

Un estudio analizó el efecto de una carta dirigida a médicos de atención primaria que atendían pacientes con somatizaciones aconsejando las vistas frecuentes, la mejor utilización de los recursos y el reconocimiento de los síntomas en conjunto con el paciente. Esta intervención, comparada con los resultados de un grupo control, mostró mejoría en los síntomas de los pacientes afectados y menor utilización de recursos ${ }^{21}$.

\section{Como hacer la derivación}

Una derivación bien hecha puede ayudar a profundizar el diagnóstico de una comorbilidad psiquiátrica. Sin embargo, los pacientes con estos trastornos son habitualmente escépticos respecto a una derivación a salud mental ya que no asocian sus síntomas con problemas de este tipo. Por lo tanto, una derivación apresurada o forzada puede resultar para el paciente una "acusación" de que sus síntomas son "imaginarios"22. El médico de atención primaria debe "trabajar la derivación" y de allí la importancia de hacerlo en forma colaborativa. Ver figura 2.

\section{Psicoterapia}

Existen pocos estudios en atención primaria que hayan evaluado el efecto de la psicoterapia sobre la somatizaciones. Morris y $\mathrm{col}^{23}{ }^{24}$. elaboraron un programa de entrenamiento para médicos de atención primaria con el objetivo de identificar aquellos pacientes con somatización que tuvieran depresión encubierta y observaron que aquellos que pudieron reconocer la depresión tuvieron mejorías y redujeron los costos en salud.

Es difícil la investigación clínica en psicoterapia, lo que en parte se debe a la dificultad para reproducir las intervenciones y categorizar a los pacientes. Sin embargo, existen estudios publicados sobre los resultados de la implementación de psicoterapias de tipo cognitivoconductual a través de estudios aleatorizados y con comparaciones con grupos controles.

Estas técnicas buscan desacondicionar a los pacientes, identificando sistemas de creencias y asunciones sobre enfermedades que puedan ser disfuncionales, modificar conductas adaptativas que perpetúen los síntomas (ej. consultas a médicos o búsqueda constante de reaseguro). Algunos programas incluyen técnicas de relajación, meditación y actividades aeróbicas. La psicoterapia es interactiva y se realiza tanto en forma individual como grupal; se trabaja con material escrito, videos y grupos de discusión en los que se discute sobre concepciones de salud y enfermedad. Se prescriben tareas como "deberes" para realizar en la casa entre las sesiones, en las que los pacientes tienen que escribir pensamientos o sensaciones relacionados con la aparición de los síntomas ${ }^{25}$.

Los ensayos clínicos que utilizaron como base estas técnicas mostraron, en general, mejorías de los síntomas y en algunos casos disminución de los costos en la utilización de los recursos. Casi todos los estudios incluyeron pocos pacientes y el tiempo de seguimiento fue reducido. Algunos especialistas argumentan que, en realidad estos programas son más una forma de psicoeducación que de psicoterapia. Una revisión sistemática que encontró 28 estudios de este tipo que incluyeron un total de 1600 pacientes, demostró mejoría con la intervención en el $70 \%$ de los mismos ${ }^{26}$. En cuanto a los pocos estudios con técnicas psicodinámicas, un estudio controlado realizado en España mostró que la terapia familiar breve de orientación sistémica es efectiva en reducir los síntomas y en alcanzar los objetivos establecidos durante la primera. Lo interesante de esta experiencia es que no fue necesaria una derivación fuera del equipo de atención primaria y tampoco que el paciente tuviera que aceptar una explicación psicológica para sus síntomas ${ }^{27}$. 
Otros dos estudios que emplearon técnicas psicodinámicas breves fueron realizados en pacientes con colon irritable refractario al tratamiento médico. Los dos fueron realizados en ámbitos de atención secundaria y luego de un seguimiento de un año mostraron significativas diferencias síntomáticas con respecto al grupo control $^{28}$.

\section{Tratamiento psicofarmacológico}

Los trastornos psiquiátricos que se asocian a somatizaciones, específicamente ansiedad y depresión responden satisfactoriamente al tratamiento farmacológico. Por este motivo, como señalamos anteriormente, buscarlos es una parte fundamental de la evaluación.

En general, este tipo de paciente tiende a padecer más efectos adversos y a desarrollar nuevos síntomas, por lo que es conveniente comenzar con bajas dosis. La prescripción debe ser realizada en conjunto y de acuerdo con el paciente y dado que los resultados producen cierta mejoría pero ésta no es espectacular y puede demorar, no conviene insistir cuando éste sea escéptico o pesimista al respecto. Como estos pacientes carecen del componente de tristeza de la depresión, el término antidepresivo puede no ser resultar muy útil, por lo que se recomienda utilizar otro nombre para dichos fármacos. Sin embargo, como los envases que los contienen, anuncian que se trata de un medicamento antidepresivo, conviene adelantarse a dicho hallazgo dándole previamente una explicación al paciente como por ejemplo: "...este medicamento se utiliza frecuentemente para la depresión por lo que muchos lo llaman antidepresivo, sin embargo en usted lo vamos a utilizar porque ha también demostrado producir alivio en los síntomas que menciona...".

Una revisión de tratamiento de pacientes con desórdenes somatomorfos reveló mejoría en aquellos tratados con IRSS, especialmente en los trastornos relacionados con el subgrupo de los síntomas obsesivos como hipocondría ${ }^{29}$. Por este motivo, como ya se demostró en los pacientes con trastorno obsesivo compulsivo, estos pacientes responden a dosis más altas de antidepresivos, por ejemplo con fluoxetina se intenta alcanzar $80 \mathrm{mg}$.

Un muy prolijo metanálisis en pacientes con somatización evaluó ensayos clínicos aleatorizados a través de la mejoría funcional global, puntajes de síntomas o severidad del dolor ${ }^{30}$. Ver cuadro 6 . Los resultados mostraron una clara mejoría en el grupo que recibió antidepresivos, requiriéndose tratar cuatro pacientes (IC95\%: 3 - 7) para que mejore un paciente en al menos una de las áreas mencionadas. Sin embargo, nuevamente la calidad metodológica de los estudios era dispar y resultó difícil categorizar los distintos síndromes.

Cuadro 6: evidencia sobre el uso de antidepresivos para pacientes con síntomas o síndromes somáticos no explicados.

\begin{tabular}{|c|c|c|c|c|c|c|}
\hline & Tricíclieos & IRSS & $\begin{array}{c}\text { Anti } \\
\text { serotonínico }\end{array}$ & Otros & $\begin{array}{c}\text { Galidad } \\
\text { (máximo 8) }\end{array}$ & OR ([L95\%) \\
\hline Cefalea crónica & 21 & 8 & 23 & - & 4,6 & $3,4(2,7-4,4)$ \\
\hline Fibromialgia & 12 & 4 & - & 3 & 5,8 & $5,1(3,1-8,5)$ \\
\hline Sntomas gastrointestinales & 11 & - & 3 & - & 4,1 & $4,4(2,5-7,7)$ \\
\hline Dolor idiopático & 8 & 2 & 2 & - & 4,2 & $2,0(1,4 \cdot 2,8)$ \\
\hline Tinnitus & 2 & - & - & - & 4,0 & - \\
\hline Fatiga crónica & - & 2 & - & - & 4,0 & - \\
\hline Todos & 54 & 16 & 28 & 3 & 4,8 & $3,4(2,6-4,3)$ \\
\hline
\end{tabular}

IRSS= inhibidores de la recaptación de la serotonina. Resumido de O’Malley $P G$, Jackson JL, Santoro $\mathrm{J}$ et al. Antidepressant therapy for unexplained symptoms and symptom syndromes. J Fam Pract 1999; 48:980-90.

Recibido el 23/11/06 y aceptado el 30/12/06

\section{Referencias}

1. Kroenke K, Mangeslford AD. Common symptoms in ambulatory care: incidence, evaluation, therapy and outcome. Am J Med. 1989;86:262-266

2. Smith GR, Monson Ra, Ray DC. Patients with multiple unexplained symtoms. Their characteristics, functional health and health care utilization. Arch Intern Med 1986;1446:69-72

3. Hartz AJ, Noyes R, Bentler SE, Damiano PC et al. Unexplained symptoms in primary care: Perspectives of Doctors and Patients. Gen Hosp Psychiatry 2000; 22:144-152

4. Lipowsky ZJ. Somatization: The concept and its clinical applications. Am J Psychiatry 1988;145:1358-68

5. Wessely S, Nimnuam M, Sharpe M. Functional somatic syndromes: one or many. Lancet 1999; 354: 936-039.

6. Manual Diagnostico y Estadístico de los Trastornos Mentales, Cuarta Edición 1994.

7. Epstein R, Quill T. Somatization reconsidered. Arch Intern Med 1999;159:215-222

8. Deary I. A taxonomy of medically unexplained symptoms. J Psychosom Res 1999; 47:51-59

9. Burton C. Beyond somatization: a review of the understanding and treatment of medically unexplained physical symptoms. Br J Gen Pract 2003; 53:233-241

10. Servan- Shreiber D, Kolb N, Tabas G: Practical diagnosis of somatizing patients. Am Fam Phys 2000; 61:1073-8

11. Richardson R, Engel Ch. Evaluation and management of medically unexplained physical symptoms. The Neurologist 2004;10: 18-30

12. Kroenke K, Spitzer RL, Williams JB, et al. Physical symptoms in primary care. Predictors of psychiatric disorders and functional impairment. Arch Family Med 1994:9:774-9

13. Simon G, Von Korff M, Picinelli M, et al. An international study of the relation between somatic symptoms and depression. N Eng J Med 1999; 341: 1329-35

14. Barsky AJ. A 37-year old man with multiple somatic compliants. JAMA 1997; 278:673-9

15. Mc.Daniel S, Campbell T, Seaburn D. Orientación Familiar en Atención Primaria. Editorial Springer Ibérica, Barcelona 1998

16. Donovan $\mathrm{Jl}$, Blake RD, Qualitative study of interpretation of reasssurance among patients attending rheumatology clinics: just a touch of arthritis doctor? BMJ $2001320 ; 541: 44$

17. Engel GL. The need for a new medical model: A challange for biomedicine. Science 1977,196:129-136

18. Mc.Daniel S, Hepworth J, Doherty W. Medical Family Therapy. A biopsychosocial approach to families with health problems 1992. New York: Basic Books

19. Asen E, Thompson P. Intervención familiar. Guía práctica para profesionales de la salud. Editorial Paidos 1997

20. Liebermann JA. BATHE: an aproach to the interview process in the primary care setting. J Clin Psychiatry 1997; 588 suppl3 $: 3-8$

21. Smith GR, Monson RA, Ray DC. Psychiatric consultation in somatization disorder. A randomized controlled study. N Engl J Med 1986;314:1407-1

22. Smith R, Lein C, Collins C, et al. Treating patients with medically unexplined symptoms in primary care. J Gen Intern Med 2003;18.478-48

23. Morriss RK, Gask L, Ronalds C, et al. Clinical and patients satisfaction outcomes of a new treatment for somatised mental disorder taught to GPs. Br J Gen Pract 1999; 49:263-267

24. Morriss RK, Gask L, Ronalds C, et al. Cost effectivness of a new treatment for somatised mental disorder taught to GPs. Fam Pract 1999; 15:119.125

25. Kroenke K, Swindle R. Cognitive behavioral therapy for somatization and symptom syndromes: a critical review of controlled clinical trials. Psychoter Psychosom 2000; 69: 205-215

26. Raine R, Haines A, Sensky T, et al. Systematic review of mental health interventions for patients with common somatic symptoms. BMJ 2002; 325

27. Real Perez M, Rodriguez Arias Palomo JL, Cagigas Viadero J, et al. Terapia Familiar Breve: Una opción para el tratamiento de trastornos somatomorfos en atención primaria. Aten Primaria 1996; 17:241-246

28. Guthire E, Creed F, Dawson D et al. A randomised controlled trial of psychoterapy in patients with refractory iirritable bowel syndrome. Br J Psychiatry 1993;163:315-321

29. Fallon B. Pharmacotherapy of somatoform disorders. J Psychosom Res 2004; 56:455-460

30. O'Malley PG, Jackson JL, Santoro J et al. Antidepressant therapy for unexplained symptoms and symptom syndromes. J Fam Pract 1999; 48:980-90 